\title{
Effects of Proprioceptive Neuromuscular Facilitation and Treadmill Training on the Balance and Walking Ability of Stroke Patients
}

\author{
Chang-Heon Kim', Yong-Nam Kim² \\ 'Department of Physical Therapy, Graduate School, Nambu University, Gwangju; ${ }^{2}$ Department of Physical Therapy, Nambu University, Gwangju, \\ Korea
}

Purpose: The purpose of this study was to investigate the effects of combined training using proprioceptive neuromuscular facilitation (PNF) patterns and treadmills on the balance and walking ability of stroke patients.

Methods: Twenty-three stroke patients were randomized into a control group $(n=11)$, receiving only treadmill training and an experimental group $(n=12)$ receiving combined training. The use of both PNF exercise and treadmill were implemented in the combined training. Interventions were performed 5 times a week for 6 weeks. Balance ability was measured by a timed up and go (TUG) test. Walking ability was measured by a 10-meter walk test (10MWT) and a 6-minute walk test (6MWT). A paired t-test was used to compare differences between pre- and post-intervention and independent t-tests were used to compare between groups.

Results: Changes in TUG, 10MWT, and 6MWT before and after interventions were significantly different for both the experimental group and the control group $(p<0.05)$. In addition, within-group changes in the TUG, 10MWT, and 6MWT were more effective in the experimental group than in the control group $(p<0.05)$.

Conclusion: Combined training using PNF techniques and treadmills may be useful in improving the balance and walking ability of stroke patients.

Keywords: Balance ability, PNF, Stroke, Walking ability

\section{INTRODUCTION}

Stroke is a high incidence neurological disorder caused by an interruption of blood flow in the brain. ${ }^{1}$ Depending on the degree of the injury and the area of the brain affected, a stroke can affect consciousness, sensory perception, language, cognitive skills, or motor skills. ${ }^{2}$ In addition, abnormal patterns coordinating muscle tone, muscle timing, and movement resulting from a stroke can affect a person's balance and walking ability. ${ }^{3}$

Losing one's mobility is the greatest loss for stroke patients. ${ }^{4} \mathrm{Re}-$ covering the ability to walk is an important rehabilitation goal ${ }^{5}$ and is one of the greatest concerns for the patient, family, and therapist. ${ }^{6}$ However, most stroke patients have a slower gait cycle because of the difficulty in moving their affected extremity ${ }^{7}$ and thus, find it chal-

Received May 8, 2018 Revised Jun 8, 2018

Accepted Jun 30, 2018

Corresponding author Yong-Nam Kim

E-mail kyn0231@nambu.ac.kr lenging to maintain balance when walking due to their asymmetric step and shorter stride. ${ }^{8}$ Balance is the ability to maintain one's center of gravity within the base of support and to control proper body positioning by responding to various external environments. ${ }^{9}$ The ability to maintain one's balance while walking is another important rehabilitation goal. ${ }^{10}$ Balance ability can be divided into static balance, which allows a steady stand on a fixed support surface, and dynamic balance, which can be sustained when there is external stimulation and agitation of the support surface. Dynamic balance is an essential factor in walking. ${ }^{11}$

PNF is a technique that improves movement function by stimulating muscles, tendons, and joints, and increases muscle power, flexibility, and balance. ${ }^{12}$ It has been used as an intervention in musculoskeletal disorders of muscles, bones, and joints and in central

Copylight (C2018 The Korea Society of Physical Therapy

This is an Open Access article distribute under the terms of the Creative Commons Attribution Non-commercial License (Http:// creativecommons.org/license/by-nc/4.o.) which permits unrestricted non-commercial use, distribution, and reproduction in any medium, provided the original work is properly cited. 
nervous system disorders resulting from stroke. ${ }^{13-17}$ The basic principles used in PNF resulted in more muscle power when applying various patterns at the same time, and the use of combination patterns improved the proprioception, power, and stability of the trunk. ${ }^{18}$ Bobath ${ }^{19}$ noted that the pelvis is the key point for both leg control and gait pattern. Wang ${ }^{20}$ showed that PNF pelvic exercises resulted in changes in walking speed and rate in stroke patients. Trueblood et al. ${ }^{21}$ applied the PNF pelvic exercise to examine the effect of pelvic movement on the walking ability of stroke patients. Kim et al. ${ }^{22}$ when applying PNF pelvic and lower extremity exercises, showed a change in the balance ability of stroke patients.

The use of a treadmill is more effective than ground gait training. It reduces rigidity and improves the walking ability of stroke patients by enabling symmetrical walking while in a weight bearing state. ${ }^{23}$ A treadmill also constantly stimulates the calf muscles, which helps to improve balance and walking ability. ${ }^{24}$ Based on the central pattern generator theory, a treadmill activates afferent inputs by spinal level neurons and serves as a walking control for limb movement, weight shift, and posture alignment. ${ }^{25}$

The pelvis and scapula are key points for the control of gait pattern. ${ }^{19}$ Previous studies have been applied only to the pelvis ${ }^{20,21,26}$ or patterns of coordinated exercises involving the upper and lower extremities. ${ }^{27,28}$ The purpose of this study was to investigate the effect of the combination of PNF and treadmill training on the balance and walking ability of stroke patients.

\section{RESEARCH METHOD}

\section{Study design}

This study was a single-blind randomized clinical trial that included 23 patients with hemiplegia due to stroke. The patients were randomly assigned to a treadmill training group $(\mathrm{n}=11)$ and a combined training group $(\mathrm{n}=12)$.

Timed up-and-go test (TUG) time, 10-m walk test (10MWT) score, and 6-minute walk test (6MWT) score were identified as the primary outcomes.

\section{Subjects}

This study was conducted on 23 patients at hospital D in city J for 6 weeks in January and February 2017. The subjects were inpatients aged 55 to 65 years, who had been diagnosed with hemiplegia due
Table 1. General characteristics of study subjects

$(n=23)$

\begin{tabular}{lccc}
\hline & $\begin{array}{c}\text { Experimental } \\
\text { group }(n=12)\end{array}$ & $\begin{array}{c}\text { Control } \\
\text { group }(n=11)\end{array}$ & $p$ \\
\hline Sex (male/female) & $7 / 5$ & $7 / 4$ & 1.00 \\
Age (year) & $60.75 \pm 3.11$ & $60.64 \pm 3.41$ & 0.33 \\
Height (cm) & $165.08 \pm 7.83$ & $165.36 \pm 7.74$ & 0.32 \\
Weight (kg) & $65.58 \pm 8.84$ & $64.82 \pm 7.48$ & 0.72 \\
Affected side (right/left) & $8 / 4$ & $7 / 4$ & 1.00 \\
Stroke type (infarction/hemorrhage) & $11 / 1$ & $10 / 1$ & 1.00 \\
Onset time after stroke (month) & $19.67 \pm 3.89$ & $19.09 \pm 3.86$ & 0.26 \\
MMSE-K (score) & $26.50 \pm 2.07$ & $27.64 \pm 2.06$ & 0.38 \\
\hline
\end{tabular}

Values are presented as mean \pm standard deviation.

MMSE-K: mini mental state examination-Korea, TUG: timed up and go test, 10MWT: 10-meter walking test, 6MWT: 6-minutes walking test.

to stroke within the last 1 to 2 years. Inclusion criteria were: being able to independently walk $10 \mathrm{~m}$, obtaining a score of 24 or greater on the mini mental state examination-Korea (MMSE-K) in order to be able to understand and follow the researcher's instructions, and signing a consent form indicating that they fully understood the goals and procedures of this study. They were randomized into a control group $(\mathrm{n}=11)$, receiving only treadmill training, and an experimental group $(\mathrm{n}=12)$, receiving combined training (Table 1$)$.

\section{Measurements}

\section{1) TUG}

TUG is a simple test that can quickly measure mobility and balance with an intra-rater and inter-rater reliability correlation coefficient of $r=0.98-0.99$. Beginning with a 'start' command, TUG measured the time that the subject took to rise from a 46-cm height armchair, walk $3 \mathrm{~m}$, turn around as fast as possible in the direction of the affected side, walk back to the chair, and sit down. ${ }^{29}$

\section{2) 10MWT}

The 10MWT is a measure of one's walking ability with an intra-rater and inter-rater reliability correlation coefficient of $r=0.95-0.96 .^{30}$ Beginning with a 'start' command, the 10MWT measured the time required for the subject to walk $10 \mathrm{~m}$ on a course that was a total distance of $14 \mathrm{~m}$. Tape, at $2 \mathrm{~m}$ and $12 \mathrm{~m}$, indicated the start and end of the 10-m walking distance. The first and last $2 \mathrm{~m}$ of the course were used for acceleration and deceleration and were not timed. ${ }^{31}$

\section{3) $6 \mathrm{MWT}$}

The 6MWT measures walking endurance ${ }^{32}$ with an intra-rater and 
inter-rater reliability correlation coefficient of $r=0.91 .{ }^{33}$ The $6 \mathrm{MWT}$ displays a starting point and a halfway point with a total distance of $20 \mathrm{~m}$ on a flat floor with the course being repeated for a total of 6 minutes. The entire walking distance, including the number of repetitions between the starting point and the halfway point, was measured in meters $(\mathrm{m})$.

\section{Training program}

In this study, the 40-minute training programs for both groups occurred 5 times a week for 6 weeks, with 5 minutes being allocated for warm up, 30 minutes devoted to the main exercise, and 5 minutes for cool down.

The training program was applied to: 1) overload, 2) progressive, 3) individual, 4) continue, and 5) reversibility.

\section{1) Treadmill training program}

The treadmill training consisted of warm-up, treadmill, and cool down. Warm-up and cool down had a rhythmic initiation in scapular and pelvic patterns in a side-lying position. Warm-up and cool down were applied for 5 minutes to prevent injury. Using only a treadmill, the training intensity was set at 11-15 using the Borg rating of perceived exertion (RPE) scale. ${ }^{34}$ Treadmill exercise was performed for 30 minutes to facilitate movement of the affected side.

\section{2) Combined training program}

The use of both PNF exercise and treadmill were implemented in the combined training program. In the PNF concept, therapists performed diametrical scapular and pelvic motion patterns using basic physical therapy principles, procedures, and techniques. The combined training consisted of warm-up, PNF, treadmill, and cool down. Warm-up and cool down had a rhythmic initiation in scapu-

Table 2. The comparison of variable on pre and post in group

\begin{tabular}{llcccc}
\hline & \multicolumn{1}{c}{ Group } & \multicolumn{1}{c}{ Pre } & Post & $\mathrm{t}$ & $\mathrm{p}$ \\
\hline TUG (sec) & Experimental & $16.18 \pm 1.07$ & $12.63 \pm 1.58$ & 12.03 & $0.00^{*}$ \\
& Control & $16.97 \pm 1.98$ & $14.95 \pm 2.31$ & 6.73 & $0.00^{*}$ \\
\multirow{2}{*}{ 10MWT (sec) } & Experimental & $15.95 \pm 2.51$ & $11.69 \pm 1.23$ & 6.80 & $0.00^{*}$ \\
& Control & $16.33 \pm 2.02$ & $14.26 \pm 2.35$ & 7.44 & $0.00^{*}$ \\
6MWT (m) & Experimental & $291.42 \pm 37.82$ & $347.92 \pm 24.50$ & -7.75 & $0.00^{*}$ \\
& Control & $294.27 \pm 35.05$ & $312.73 \pm 33.57$ & -9.39 & $0.00^{*}$ \\
\hline
\end{tabular}

Values are presented as mean \pm standard deviation

TUG: timed up and go test, 10MWT: 10-meter walking test, 6MWT: 6-minutes walking test.

${ }^{*} p<0.05$. lar and pelvic patterns in a side-lying position. Warm-up and cool down were applied for 5 minutes to prevent injury. PNF exercise involved hold-relax, contract-relax, and dynamic reversal in symmetrical reciprocals combined with scapular and pelvic patterns in sidelying, side-elbow, and quadrupedal positions. PNF was applied for 15 minutes to facilitate movement of the affected side. By using only a treadmill, the training intensity was set at a RPE of 11-15. Treadmill exercise was performed for 15 minutes to facilitate movement of the affected side.

\section{Data analysis}

Statistical analyses were performed using the SPSS/PC for Windows version 18. The Shapiro-Wilk test was used to check the normal distribution of the data. The paired t-test was performed to compare differences in balance ability and walking ability within groups before and after interventions. The independent t-test was performed in order to compare the differences between the experimental and control groups. The level of significance was set at $\alpha=0.05$.

\section{RESULTS}

\section{Comparison of TUG changes}

Within-group changes in the TUG test were significantly different, in both the experimental group $(\mathrm{p}<0.05)$ and the control group $(\mathrm{p}<0.05)$ (Table 2$)$, with the experimental group showing greater improvement than the control group $(\mathrm{p}<0.05)$ (Table 3$)$.

\section{Comparison of 10MWT changes}

Within-group changes in the 10MWT were significantly different, in both the experimental group $(\mathrm{p}<0.05)$ and the control group $(\mathrm{p}<0.05)$ (Table 2$)$, with the experimental group showing greater improvement than the control group $(\mathrm{p}<0.05)$ (Table 3$)$.

Table 3. The comparison of changes in variables between post-test in both group

\begin{tabular}{lcccc}
\hline & Experimental & Control & $\mathrm{t}$ & $\mathrm{p}$ \\
\hline TUG (sec) & $12.63 \pm 1.58$ & $14.95 \pm 2.31$ & -2.83 & $0.01^{*}$ \\
1OMWT (sec) & $11.69 \pm 1.23$ & $14.26 \pm 2.35$ & -3.33 & $0.00^{*}$ \\
6MWT (m) & $347.92 \pm 24.50$ & $312.73 \pm 33.57$ & 2.89 & $0.01 *$ \\
\hline
\end{tabular}

Values are presented as mean \pm standard deviation.

TUG: timed up and go test, 10MWT: 10-meter walking test, 6MWT: 6-minutes walking test.

$* p<0.05$. 


\section{Comparison of 6MWT changes}

Within-group changes in the 6MWT were significantly different, in both the experimental group $(\mathrm{p}<0.05)$ and the control group $(p<0.05)$ (Table 2), with the experimental group showing greater improvement than the control group $(\mathrm{p}<0.05)$ (Table 3$)$.

\section{DISCUSSION}

In this study, 23 patients with hemiplegia due to stroke were randomly sorted into a treadmill training group $(\mathrm{n}=11)$ and a combined training group $(n=12)$, with the change in balance and walking ability evaluated after 6 weeks of intervention.

Initially, therapists performed PNF symmetrical reciprocal combined scapular and pelvic patterns on the unaffected side of patients who were then asked to actively repeat each movement on both sides, thereby learning the motor skill through active assistance and resistance. Bobath ${ }^{19}$ performed interventions with the trunk or pelvis to facilitate the normal gait pattern of the hemiplegic patient, and found that the key point of movement control is in the proximal part of the body. In addition, interventions to stabilize the trunk of the body were implemented to reduce the atrophy of muscle tone and improve movement in patients with central nervous system injuries.

In this study, we used TUG, 10MWT, and 6MWT, all with high intra-rater reliability, to evaluate the balance and walking ability of hemiplegic patients due to stroke. ${ }^{29,30,33}$ The findings of this study may be helpful in predicting the stroke patients' balance and walking abilities and their eventual return to independence. In this study, the TUG test was used to evaluate the dynamic and static balance abilities of stroke patients. The 10MWT was used to determine the change in gait speed of the stroke patients according to intervention for scapular and pelvic movements. The 6MWT was used to evaluate the change in gait endurance of the stroke patients according to scapular and pelvic movements, and the treadmill training.

Trueblood et al. ${ }^{21}$ reported improvement in walking ability after application of pelvic PNF-based exercise regimes on the affected side in 20 hemiplegic patients due to stroke. Wang ${ }^{20}$ identified changes in walking ability by applying rhythmic initiation, dynamic reversal, and slow reversal techniques to pelvic PNF-based exercise regimes by dividing hemiplegic patients into acute and chronic groups. As a result, there was an improvement early in the acute group, but no corresponding change in the chronic group. However, over time, changes did occur. Kim et al. ${ }^{22}$ applied a pelvic and lower extremity PNF exercise pattern with rhythmic initiation techniques to the affected side of hemiplegic patients, and obtained a positive change in balance and activities associated with daily living.

In this study, we applied a symmetrical reciprocal combined scapular and pelvic PNF exercise pattern to the unaffected side of hemiplegic patients to eliminate their fear of pain associated with movement in comparison to previous studies. The combination of sympathetic reciprocals through scapular and pelvic exercises initiated on the unaffected side, transferred new neurological innervations to the patients' affected side and in combination with the positive effects of treadmill walking, resulted in their ability to maintain balance and proper gait cycles.

Kim et al. ${ }^{26}$ obtained a positive effect for balance and walking ability after applying the limb pattern PNF to hemiplegic stroke patients. $\mathrm{Lim}^{27}$ obtained a positive effect for balance and walking ability by applying the sprint and skate PNF patterns to hemiplegic patients. These studies wish to suggest a more positive outcome using these PNF patterns, compared to the present study by more closely approximating the gait pattern. However, Bobath ${ }^{19}$ suggested that development of normal trunk control ability is essential and that interventions to stabilize the trunk, reducing muscle atrophy, and allowing for free limb movement, should precede additional interventions such as the scapular and pelvic PNF patterns.

In this study, both the combined and treadmill training groups showed improvement in balance and walking ability. The symmetrical reciprocal combined scapular and pelvic PNF patterns reestablished motor control for the gait pattern. Treadmill exercises with increased trunk stability may have had a positive impact on balance and walking ability.

This study was effective in improving balance and walking ability by applying PNF exercises and treadmill training programs to hemiplegic stoke patients, therefore, we consider this combined program to be useful for these patients.

\section{REFERENCES}

1. Bae SS, Lee JH. The study of clinical pictures and received rehabilitation service patterns of stroke patients. J Kor Soc Phys Ther. 2001;13(3):799814.

2. Jørgensen HS, Nakayama H, Raaschou HO et al. Recovery of walking function in stroke patients: the copenhagen stroke study. Arch Phys Med 
Rehabil. 1995;76(1):27-32.

3. Patterson KK, Parafianowicz I, Danells CJ et al. Gait asymmetry in community-ambulating stroke survivors. Arch Phys Med Rehabil. 2008; 89(2):304-10.

4. Mumma CM. Perceived losses following stroke. Rehabil Nurs J. 2000; 25(5):192-5.

5. Werner C, Bardeleben A, Mauritz KH et al. Treadmill training with partial body weight support and physiotherapy in stroke patients: a preliminary comparison. Eur J Neurol. 2002;9(6):639-44.

6. Bohannon RW, Andrews AW, Smith MB. Rehabilitation goals of patients with hemiplegia. Int J Rehabil Res. 1988;11(2):181-4.

7. Ryerson S, Levit K. Functional movement reeducation: a contemporary model for stroke rehabilitation. Philadelphia, Churchill Livingstone, 1997.

8. Carr J, Shepherd R. Neurological rehabilitation: optimizing motor performance. New York, Churchill Livingstone, 2010.

9. Horak FB. Clinical assessment of balance disorders. Gait Posture. 1997; 6(1):76-84.

10. Keenan MA, Perry J, Jordan C. Factors affecting balance and ambulation following stroke. Clin Orthop Relat Res. 1984;1(182):165-71.

11. Rode G, Tiliket C, Boisson D. Predominance of postural imbalance in left hemiparetic patients. Scand J Rehabil Med. 1997;29(1):11-6.

12. Klein DA, Stone WJ, Phillips WT et al. PNF training and physical function in assisted-living older adults. J Aging Phys Act. 2002;10(4):476-88.

13. Kim BR, Lee HJ. Effects of proprioceptive neuromuscular facilitationbased abdominal muscle strengthening training on pulmonary function, pain, and functional disability index in chronic low back pain patients. J Exerc Rehabil. 2017;13(4):486-90.

14. Jeong WM, Kim BR. The effects of proprioceptive neuromuscular facilitation exercise on the pain and functional disability index of patients with chronic lower back pain. PNF \& Movement. 2017;15(2):195-200.

15. Kim CH, Kim BR. The effects of abdominal strength training using proprioceptive neuromuscular facilitation on the balance ability and pain of patients with chronic lower back pain. PNF \& Movement. 2017;15(2):1418.

16. Song MS, Kim BR, Kim CH et al. A case report of a proprioceptive neuromuscular facilitation intervention strategy applied with an ICF tool in a patient with anterior cruciate ligament reconstruction. PNF \& Movement. 2017;15(1):1-11.

17. Jeong WM, Kim BR, Kang MG. Effect of treadmill training and proprioceptive neuromuscular facilitation lower leg taping on balance and gait ability in stroke patients. PNF \& Movement. 2016;14(2):83-91.

18. Dietz B. Let's sprint, let's skate: innovationen im PNF-Konzept. Heidel- berg, Springer, 2009.

19. Bobath B. Adult hemiplegia: evaluation and treatment. Oxford, Butterworth Heinemann, 1990.

20. Wang RY. Effect of proprioceptive neuromuscular facilitation on the gait of patients with hemiplegia of long and short duration. Phys Ther. 1994; 74(12):1108-15.

21. Trueblood PR, Walker JM, Perry J et al. Pelvic exercise and gait in hemiplegia. Phys Ther. 1989;69(1):18-26.

22. Kim EK, Lee DK, Kim YM. Effects of aquatic PNF lower extremity patterns on balance and ADL of stroke patients. J Phys Ther Sci. 2015;27(1): 213-5.

23. Hesse S, Werner C, Bardeleben A et al. Body weight-supported treadmill training after stroke. Curr Atheroscler Rep. 2001;3(4):287-94.

24. Hesse S. Treadmill training with partial body weight support in hemiparetic patients-further research needed. Neurorehabil Neural Repair. 1999;13(3):179-81.

25. Dietz V. Body weight supported gait training: from laboratory to clinical setting. Brain Res Bull. 2008;76(5):459-63.

26. Kim K, Lee DK, Jung SI. Effect of coordination movement using the PNF pattern underwater on the balance and gait of stroke patients. J Phys Ther Sci. 2015;27(12):3699-701.

27. Lim CG. The effects of proprioceptive neuromuscular facilitation (PNF) pattern exercise using the sprinter and the skater on balance and gait function in the stroke patients. J Kor Soc Phys Ther. 2014;26(4):249-56.

28. Kim BR, Bang DH, Bong SY. The effect of coordinative pattern exercise of upper and lower extremities use harness for walking ability and balance ability in chronic stroke patients. PNF \& Movement. 2015;13(3):127-34.

29. Podsiadlo D, Richardson S. The timed "Up \& Go": a test of basic functional mobility for frail elderly persons. J Am Geriatr Soc. 1991;39(2):1428.

30. Van Loo M, Moseley A, Bosman J et al. Test-re-test reliability of walking speed, step length and step width measurement after traumatic brain injury: a pilot study. Brain Inj. 2004;18(10):1041-8.

31. Walker C, Brouwer BJ, Culham EG. Use of visual feedback in retraining balance following acute stroke. Phys Ther. 2000;80(9):886-95.

32. Swisher AK, Goldfarb AH. Use of the six-minute walk/run test to predict peak oxygen consumption in older adults. Cardiopulmonary Phys Ther J. 1998;9(3):3-5.

33. Mossberg KA. Reliability of a timed walk test in persons with acquired brain injury. Am J Phys Med Rehabil. 2003;82(5):385-90.

34. Borg GA. Psychophysical bases of perceived exertion. Med Sci Sports Exerc. 1982;14(5):377-81. 